\title{
Interface roughness induced intrasubband scattering in a quantum well under an electric field
}

\author{
G.B. Ibragimov \\ Institute of Physics, Academy of Science of the Azerbaijan, 33 H. Javid av., 370143 Baku, Azerbaijan \\ Fax: +380 (99412) 39 5961; e-mail: physic@physics.ab.az
}

\begin{abstract}
Scattering rates in the lowest subband in a quantum well are calculated for interface roughness scattering when an electric field is applied normally to the layer plane. It is found that the interface roughness scattering rate increases with increasing electric field. The electric field changes the interface roughness scattering rates drastically in thick QWs as compared with those for the zero-field case.
\end{abstract}

Keywords: interface roughness, intrasubband transition, quantum well.

Paper received 23.06.01; revised manuscript received 21.12.01; accepted for publication 05.03.02.

\section{Introduction}

There has been considerable interest in the study of intersubband transitions in a quantum well (QW) both in the presence and in the absence of an electric field applied perpendicular to the QW layer plane [1-12]. The reason causing the interest is practical device application [3-4]. In [1] proposed is a new type of infrared laser amplifier using the intersubband transition and resonant tunneling. An infrared detector in the $10 \mu \mathrm{m}$ has been proposed and fabricated [5] that relies on the intersubband transition and resonant tunneling between adjacent QWs.

Theoretical studies [6,7] on intersubband optical absorption under an electric field have pointed out an increase in the oscillator strength. In all the theories $[6,7]$ the intra- and intersubband relaxation rates come into the calculation of the absorption coefficient. Various scattering events determine the intra- and intersubband relaxation rates, of which polar optic phonon scattering has been major scattering mechanism over a considerable temperature range. The rates for such scattering have already been calculated [7]. In [8] the alloy-disorder scattering rate have been calculated for two-dimensional electrons in the lowest subband of a quantum well in an applied electric field.
It is established that interface - roughness scattering determines the low temperature mobility of two - dimensional (2D) electrons in thin QWs $[13,14]$. The mobility and relaxation rates due to interface roughness scattering in QWs have been formulated previously in the absence of an applied electric field $[15,16]$. In the present work we report similar calculations when an electric field is applied perpendicular to the QW layer, and investigate how the scattering rates are modified. As a first step, we consider intrasubband relaxation for the lowest subband.

\section{Calculations}

The electrons are assumed to be quantizied along the $\mathrm{Z}$ direction and the wave function is taken to be of the following form [2].

$$
\begin{aligned}
& \Phi(R)=\Psi(Z) \exp (i k r)=N(\beta)\left(1+\frac{\beta z}{L}\right) \Psi_{0}(z) \exp (i k r) \\
& -L / 2<z<L / 2
\end{aligned}
$$

where $\psi_{0}(Z)$ is the unperturbed ground-state wave function. The normalization factor of the electron wave function are

$$
N^{2}(\beta)=\frac{\pi^{3}}{\pi^{3}+\beta^{2}\left(\pi^{2}-8\right)}
$$

$L$ is the thickness of the well, and $k$ and $r$ are, respectively the $2 \mathrm{D}$ wave vector and position vector of the electron in the plane of free motion (X-Y plane). In Eq. (1) $b$ is variational parameter related to the electric field $F$ as in [2] 


\section{G.B. Ibragimov: Interface roughness induced intrasubband scattering...}

$\beta=\frac{2|e| F m^{*} L}{\hbar^{2}}<z^{2}>_{0} \quad, \quad \beta^{2}<z^{2}>_{0}<<L^{2}$
where $\quad<z^{2}=<\psi\left|z^{2}\right| \psi_{0}>$

The interface roughness is characterized by the height $\Delta$ and the lateral correlation length $\wedge$ of the Gaussian fluctuation. For the perturbing potential due to interface roughness we use the standard model [10,17], for which the random potential is assumed to have the Gaussian distribution and expressed as the autocorrelation function

$<V(R) V\left(R^{\prime}\right)>=$

$=V_{0}^{2} \Delta^{2} \delta(Z-L / 2) \delta\left(Z^{\prime}-L / 2\right) \exp \left(-\frac{\left(r-r^{\prime}\right)^{2}}{\Lambda^{2}}\right)$

where $\langle\ldots . . .$.$\rangle means an ensemble average, \mathrm{V}_{0}$ is the barrier height.

Using Eq. (4) we got for the square of the matrix element for roughness scattering from the $\mathrm{k}$ state to the $\mathrm{k}$ " state

$$
\begin{aligned}
& \left|M\left(K, K^{\prime}\right)\right|^{2}= \\
& =\pi \Lambda^{2} \Delta^{2} V_{0}^{2} \psi^{4}\left(\frac{L}{2}\right) \exp \left(-\frac{q^{2} \Lambda^{2}}{4}\right)
\end{aligned}
$$

where $\mathrm{q}=\mathrm{k}$ " $-\mathrm{k}$ is the $2 \mathrm{D}$ scattering wave vector and $\mathrm{q}=|q|$. Also, as the scattering is elastic

$k^{\prime}=k, q^{2}=2 k^{2}(1-\cos \theta), \cos \theta=k k^{`} / k^{2}$

The transition probability from a state $k$ to all other states $\mathrm{k}$ is then given by

$=\frac{2 \pi}{\hbar} \sum_{K^{\prime}}\left|M\left(K, K^{\prime}\right)\right|^{2} \delta\left(E_{K^{\prime}}-E_{K}\right)$

$=\frac{\pi m^{*} \Lambda^{2} \Delta^{2} V_{0}^{2} \psi^{4}(L / 2)}{\hbar^{3}} G(\Lambda, K)$

where

$G(\Lambda, K)=\frac{1}{2 \pi} \int_{0}^{2 \pi} \exp \left[-\Lambda^{2} K^{2}(1-\cos \theta) / 2\right] d \theta$

The interface roughness scattering rate is proportional to $V_{0}^{2} \psi^{4}(L / 2)$, and using [9]

$\psi(L / 2)=\left.\left(\frac{\hbar^{2}}{2 m *\left(V_{0}-E\right)}\right)^{1 / 2} \frac{d \psi}{d Z}\right|_{Z=L / 2}$

We obtain

$W_{F}=\frac{\pi^{5} \Lambda^{2} \Delta^{2} \hbar}{m^{*} L^{6}} \cdot \frac{V_{0}^{2}}{\left(V_{0}-E\right)^{2}} N^{4}(\beta)\left(1+\frac{\beta}{2}\right)^{4} \cdot G(\Lambda, K)$
As shown in Eq. (8), the scattering rate is proportional to $L^{-6}$, this means that interface roughness scattering rate is much more important for narrow wells and the interface roughness scattering limited mobility in quantum wells is proportional to $L^{6}$ [15].

For the interface roughness calculation we have not bothered to take screening into account. It seems evident that screening can only reduce an effect that is already very small.

In order to facilitate comparison, a similar expression for the transition probability or scattering rate without an applied field is needed. Thus, if we take the limit $V_{0} \rightarrow \infty$ and $\beta=0$ in Eq.(8), we may write for the scattering rate without a field, $W_{0}(K)$ as [15]

$W_{0}=\frac{\pi^{5} \Lambda^{2} \Delta^{2} \hbar}{m * L^{6}} G(\Lambda, K)$

In the limit $V_{0} \rightarrow \infty$ from Eqs (8) - (9) the ratio is expressed as

$\gamma=\frac{W_{F}(K)}{W_{0}(K)}=N^{4}(\beta)\left(1+\frac{\beta}{2}\right)^{4}$

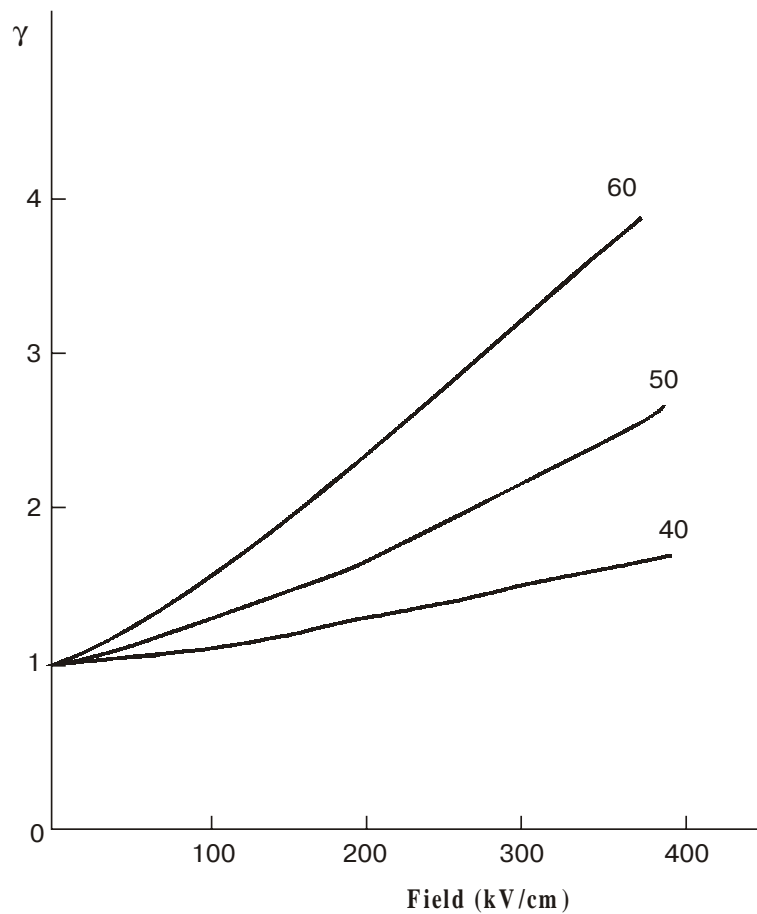

Fig. 1. Variation of scattering rate, $W_{F}(k)$, with applied electric field for a GaAs QW of 40,50 or $60 \mathrm{~A}^{0}$. The rate $W_{F}$ is normalized by the rate $W_{0}(k)$ in the absence of field, and the ratio is denoted by $\gamma$.

\section{Results}

We have calculated the scattering rates in QWs by using the parameters characteristic of GaAs. The values of the ratio $\gamma$ calculated from Eq. (10) plotted against the electric field $F$ in Fig.1. It is found from the figure that the scattering rate increases with an increase in the electric field. However, as seen from the figure, the electric field changes the interface roughness scattering rates drastically in thick QWs 


\section{G.B. Ibragimov: Interface roughness induced intrasubband scattering...}

as compared with those for zero-field case. These results predict qualitatively the trends observed [2]. Smaller electric field dependences can be explained by the fact that the wave function change due to the applied electric field of thin QWs is smaller than that thick QWs. We find that $\gamma$ is also a strong function of field and this ratio becomes as small as 1.1 to 1.5 below $100 \mathrm{kV} / \mathrm{cm}$. Another observation is that although the value for $\gamma$ is $1.5 \div 4.5$ field above $400 \mathrm{kv} / \mathrm{cm}$. In the case of polar optic phonon scattering $\gamma \approx 1.2(\mathrm{~F}=200 \mathrm{kV} / \mathrm{cm})$ [7] and for alloy-disorder scattering $\gamma \approx 4.5(\mathrm{~F}=400 \mathrm{kV} / \mathrm{cm})$ [8].

\section{Conclusion}

We have demonstrated that there is an increase of the scattering rate of electrons in the first subband of a QW, with increase in the perpendicular electric field, when the scattering is due to interface roughness. We find that $W_{F} / W_{0}$ have a stronger dependence on the well width.

\section{Acknowledgments}

The author would like to thank Prof. M.I. Aliev and Prof. F.M. Gashimzade for helpful discussions.

\section{References}

1. R.F.Kazarinov and R.A.Suris. On possibility increases electromagnetic wave in semiconductor superlattice// Fiz. Tekh. Poluprovodn. 5, pp.797-800 (1971).

2. G.Bastard, E.E.Mendez, L.L.Chang and L.Esaki. Variational calculations on a quantum well in an electric field // Phys. Rev. B 28(6), pp. 3241-3245 (1983)

3. Intersubband Transition in Quantum Wells. Ed. by E.Rosencher, B.Vinter, B.Levine. N.Y. (1992).
4. Quantum Well Intersubband Transition: Physics and Devices. Ed. by H.C.Liu, B.F.Levine, J.Y.Anderson, Kluwer Academic, Dordrecht (1994)

5. B.F.Levine, R.J.Malik, J.Walker, K.K.Choi, C.G.Bethea, D.A.Kleinman, and J.M.Vandenberg., New $10 \mu \mathrm{m}$ infrared detector using intersubband absorption in resonant tunneling GaAlAs superlattices// Appl. Phys. Lett 50, pp.1092-1094 (1987).

6. D.Ahn and S.H.Chuang J, Appl. Nonlinear intersubband optical absorption in a semiconduator quantum well // J.Appl.Phys. 62, pp. 3052-3054 (1987).

7. D.Ahn and S.H.Chuang., Electric field dependence of intrasubband polar-optical-phonon scattering in a quantum well// Phys. Rev B. 37(5), pp.2529-2535 (1988).

8. P.K.Basu, D.Raychaudhury. Alloy-disorder-induced intrasubband scattering in a quantum well under an eletric field// J.Appl.Phys. 68(7), pp.3443-3444 (1990).

9. I.Dharssi and P.N.Butcher. Interface roughness scattering in a superlattice// J. Phys: Condensed Matter 2, p. 4629-4635 (1990)

10. B.Vinter and L.Thibaudean. Bound to free state infrared absorption and selection rules in quantum wells. In: Intersubband Transition in Quantum Wells. Ed. by E.Rosencher, B.Vinter, B.Levine, p. 329. N.Y. (1992)

11. A.G.Petrov, A.Shik. Interlevel optical transitions in potential wells//Fiz.Tekh.Poluprovodn. 27(6), p. 1047 (1993).

12. G.B.Ibragimov. Free-carrier absorption in quasi-dimensional systems. Int. Conf. Opt. Semicond. OS 2000 (Ulyanovsk, 2000) p. 25

13. S.Mori and T.Ando. Electronic Properties of the semiconductor superlattice. Low temperature mobility perpendicular to the superlattice. // J. Phys. Soc. Japan 48(3), pp.865873 (1980)

14. A.Gold. Electronic transport properties of two-dimensional electron gas in a silicon quantum-well structure at low temperatures. // Phys. Rev B.35, 723 (1987).

15. H.Sakaki, T.Noda, K.Hirakawa, M.Tanaka, and T.Matsusue. Interface roughness scattering-limited electron mobility in AlAs/GaAs and $\mathrm{Ga}_{0.5} \operatorname{In}_{0.5}$ quantum wells. // Appl. Phys. Lett. 51 (23), pp.1934-1936 (1987)

16. B.R.Nag, S. Mukhopadhyay, and M.Das., Interface roughness scattering-limited electron mobility in $\mathrm{AlAs} / \mathrm{GaAs}$ and $\mathrm{Ga}_{0.5} \mathrm{In}_{0.5}$ wells. J. Appl. Phys. 86(1), pp.459-463 (1999).

17. T.Ando, A.B.Fowler and F.Stern. Electronic properties of two-dimensional systems. // Rev. Mod. Phys .54, pp.437-672 (1982). 